
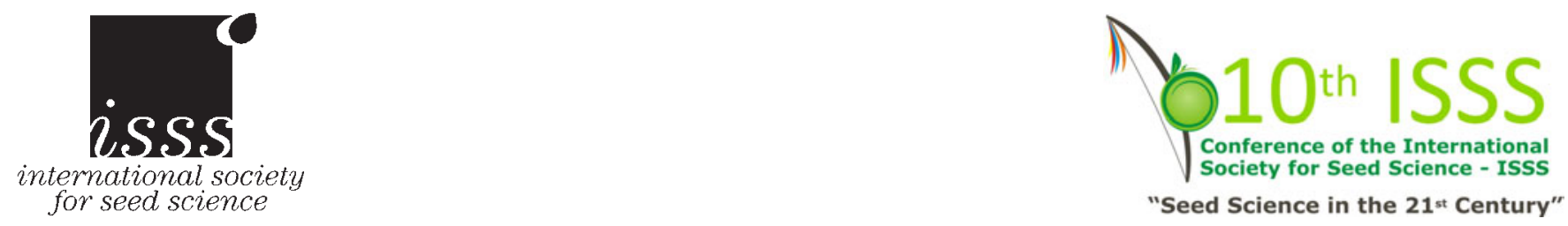

\title{
REVIEW
}

\section{Seed treatments: phytotoxicity amelioration and tracer uptake}

\author{
A.G. Taylor ${ }^{\star}$ and Y.A. Salanenka \\ Department of Horticulture, NYSAES Cornell University, Geneva, NY 14456, USA
}

(Received 26 May 2011; accepted after revision 21 September 2011)

\begin{abstract}
Seed treatments are used globally on a wide range of field, vegetable and ornamental seeds, for efficient early season control of insects and diseases. However, specific seed-treatment compounds may be phytotoxic and this phytotoxicity is most acute in laboratory germination tests. Several strategies have been developed to alleviate seed-treatment phytotoxicity that include spatial separation of the pesticide from the seed. This can be accomplished by the application of the active compounds at the end of pelleting or by using a two-pellet system, termed 'smart-pill technology'. Another approach is to detoxify or adsorb the agrochemical in a standard germination test by applying a peat medium over the seeds in a roll towel or blotter test. Many new seed-treatment chemicals have systemic activity, and the efficacy of these systemic seed treatments depends on the ability of these applied chemical compounds to be absorbed, and then transported in the developing plant. The present article describes seed-coat permeability to systemic seed treatments, examined by monitoring the movement of fluorescent tracers into intact seeds during imbibition. Two moderately lipophilic, fluorescent tracers have been used - rhodamine (ionic) and coumarin (non-ionic) - which differ mainly in electrical charge. Seed-coat permeabilities of particular species have been grouped into three categories: (1) permeable to both tracers; (2) selectively permeable to only coumarin; and (3) non-permeable to both tracers. The ability of a particular compound to diffuse through the seed coat is related to the
\end{abstract}

*Correspondence

Email: agt1@cornell.edu chemical nature of the seed-covering tissues and the physico-chemical properties of the compound applied.

Keywords: coumarin, film coat, rhodamine, seed-coat permeability, seed pellet, systemic

\section{Introduction}

Seed treatments include a wide range of materials and our discussion will focus on chemicals that reduce pest damage and/or have systemic activity. Seed-coating technologies are employed for several reasons, including the application of agrochemical seed treatments. The objectives of this paper are to review methods that reduce seed-treatment phytotoxicity and to discuss seed-coat permeability in relation to uptake of systemic compounds. The first topic is of particular interest in crop production and in seed testing. The second topic provides information on the diffusion of compounds through seed coats. A technique is proposed to determine the seed-coat permeability characteristic of any species. An understanding of seed-coat permeability and seed anatomy provides a basis for an explanation of the occurrence of seed-treatment phytotoxicity prior to radicle emergence.

\section{Reducing phytotoxicity of seed treatments}

Chemical seed treatments are used for the control of pests and diseases; however, they may have negative side effects that reduce seed germination or seedling growth. The degree of phytotoxicity depends upon the 
active ingredient, the application rate (Russell and Mussa, 1977) and the quality of the seed lot (Kuhar et al., 2002). Several strategies have been developed to alleviate seed-treatment phytotoxicity. They include: spatial or temporal separation of the pesticide from the seed. Another approach is to detoxify or adsorb the agrochemical in a standard germination test.

The first technique is to provide physical separation between the chemical and the seed using coating technologies. Two components are used to make a seed pellet: coating filler and binder (Halmer, 1999; Taylor, 2003). During the pelleting process, fillers and binders are applied, resulting in an increase in seed surface and a build-up in size. Agrochemical seed treatments can then be applied in a band in the later stages of the pelleting operation, termed 'pellet loading' (Hill, 1999), which prevents all the chemical from being absorbed into the seed at once. This technology is used on a commercial basis by the seed industry (Hill, personal communication). Another use of seed pelleting to achieve separation of active ingredients from the germinating seed is termed 'smart-pill technology'. This technology was developed for high-value, greenhouse crops (Tetteroo et al., 2009). First, a portion of seeds of the crop species is killed by radiation, then pellets are made with the non-viable seeds and the seed treatment is applied into or on the pellet of these dead seeds. Second, a high-quality seed lot of the same crop is pelleted without the phytotoxic seed treatment. A dead, treated, pelleted seed and a viable pelleted seed are sown together in the same plug. The viable seed germinates and systemically absorbs the active ingredient from the pelleted dead seed. Temporal separation is still another method of reducing phytotoxicity and may be achieved with the use of slow-release seed treatments that reduce the dosage of pesticide to the germinating seed over time (Kanampiu et al., 2009).

Seed testing is mandated to determine germination of seed lots prior to sale. Test methods were developed to provide environmental conditions that will allow for optimum exhibition of viability. In general, seeds are placed in close proximity on an inert medium for routine testing. Under these test conditions, treated seeds can have a higher concentration of active chemicals in the surrounding environment than found in the field. High concentrations of seed-treatment chemicals can negatively impact the germination rate, and increase the number of abnormal and dead seeds. Therefore, the perceived phytotoxicity observed under laboratory test conditions may be greater than actual phytotoxicity in crop production. Practical methods have been sought to ameliorate these biased conditions found in a standard germination test of treated and coated onion (Allium cepa) seeds.

Onion seed lots were pelleted or film coated with the seed-treatment insecticide cyromazine and a combination of two seed-treatment fungicides, thiram and carboxin. This seed-treatment combination significantly reduced percent germination (normal seedlings) in standard germination tests conducted on roll towels (Taylor et al., 2001). Cyromazine, a triazine compound, had a pronounced effect on impairing root development, resulting in an increase of abnormal seedlings. The application of a peat medium over roll towels reduced this injury by the absorption of excess chemical. Based on these results, and additional referee tests, the Association of Official Seed Analysts (AOSA) adopted the following rule change: AOSA Rules for Testing Seed Volume 1, Section 6.8 Special Procedures and Alternate Methods for Germination (AOSA, 2009).

\section{Seed-coat permeability in relation to uptake of systemic compounds}

Systemic uptake of active compounds was first studied on plant roots (Edgington and Peterson, 1977; Briggs et al., 1982), and the physico-chemical properties related to root uptake were examined on seed-coat permeability of selected crop seeds (Salanenka and Taylor, 2011). Fluorescent tracers and a coloured compound were used to mimic systemic seed treatments and to visualize diffusion of compounds through the seed coat. Model tracers had different chemical properties: $\log K_{\mathrm{ow}}$, electrical charge, H-bonding capacity, molecular weight and solubility. The $\log K_{\text {ow }}$ is the octan-1-ol/water coefficient of a compound (also known as $\log P$ ), and is a measure of the lipophilicity of a compound. Figure 1 illustrates the effect of $\log K_{\text {ow }}$ on systemic uptake by roots (adapted from Briggs et al., 1982), and the $\log K_{\text {ow }}$ and electrical charge of tetrazolium red, rhodamine $B$ and coumarin 151 are shown. Tetrazolium red is a water-soluble compound routinely used in seed testing, while rhodamine and coumarin both have systemic activity, but differ in electrical charge.

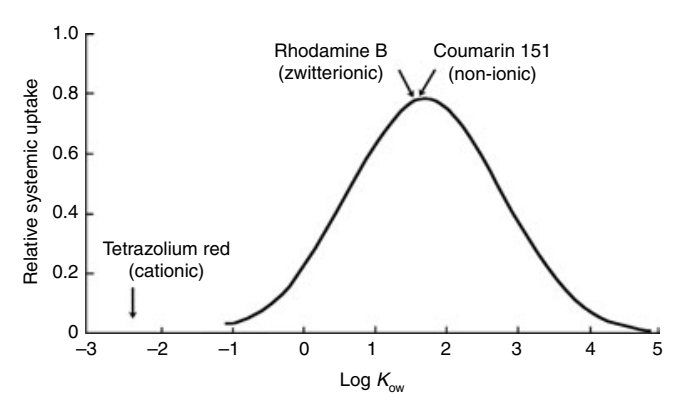

Figure 1. Relative systemic uptake of compounds by roots in relation to their $\log K_{\mathrm{ow}}$ (partition of a compound in octan-1ol/water). The $\log K_{\text {ow }}$ and electrical charge of tetrazolium red, rhodamine B and coumarin 151 are illustrated. (Adapted from Briggs et al., 1982). 
Table 1. Seed coat permeability characteristics of ten seed species representing seven plant families. Data adapted from Salanenka and Taylor $(2009,2011)$

\begin{tabular}{llll}
\hline Family & Genus species & Common name & Permeability \\
\hline Fabaceae (sub-family Faboideae) & Glycine max & Soybean & Permeable \\
& Phaseolus vulgaris & Snap bean & Permeable \\
& Pisum sativum & Pea & Permeable \\
Lilliaceae & Allium cepa & Onion & Selectively permeable \\
Poaceae & Panicum virgatum & Switchgrass & Selectively permeable \\
Solanaceae & Capsicum annuum & Pepper & Selectively permeable \\
& Solanum lycopersicum & Tomato & Selectively permeable \\
Asteraceae & Lactuca sativa & Lettuce & Non-permeable \\
Cucurbitaceae & Cucumis sativus & Cucumber & Non-permeable \\
Euphorbiaceae & Ricinus communis & Castor oil plant & Non-permeable \\
\hline
\end{tabular}

Seed-coat permeability to fluorescent tracers was studied in ten species representing seven plant families and resulted in the formation of three categories: (1) permeable; (2) selectively permeable; and (3) non-permeable (Table 1). All large-seeded legumes in the sub-family Faboideae had permeable seed coats to solute diffusion. Inorganic ions, as well as organic compounds, would pass through the permeable seed coats, and this provides the premise for electrolyte leakage as a measure of large-seeded legume seed quality (Copeland and McDonald, 2001). Selective permeability is a new category in which seed coats were observed to be permeable to only non-ionic, moderately lipophilic compounds. Seeds with selectively permeable seed coats, such as onion, tomato and peppers, have a hydrophobic semi-permeable layer in the innermost part of their seed coat (Beresniewicz et al., 1995a). The semi-permeable layer in this group would be a barrier to water-soluble compounds, including tetrazolium red, amino acids and sugars, as well as electrolytes. The inner layer of the onion seed coat is composed of cutin, while in tomato and peppers the inner layer is composed of suberin (Beresniewicz et al., 1995a). In many grass species, a semi-permeable cutinized or suberized membrane in the caryopsis integuments restricts solute diffusion (Simpson, 1990). Lettuce and cucumber seed coverings were non-permeable to solute diffusion. Lettuce embryos are surrounded by a mannan-rich endospermic layer (Halmer et al., 1975), while the envelope in cucurbits consists of a single layer of endosperm whose outer surface is covered with lipid and callose layers (Yim and Bradford, 1998).

One outcome of this research was the development of a relatively simple method to characterize the seed-coat permeability in any species by testing uptake using only rhodamine B and coumarin 151 . Both tracers are available from laboratory chemical suppliers. Positive embryo staining with coumarin 151 and rhodamine $\mathrm{B}$ indicates permeable seed coats. Positive embryo staining with coumarin 151 and negative staining for rhodamine B indicates selective permeability, while negative staining for both tracers indicates non-permeable seed coats. Collectively, determining the seed-coat permeability characteristic of a species should predict whether a particular compound is taken up during imbibition.

\section{Proposed explanation of seed-coat permeability with seed-treatment phytotoxicity}

Systemic seed-treatment compounds generally have low water solubility so they are formulated as high-percentage active ingredient liquids or dry powders that can be dispersed in water. For example, the seed-treatment formulation Gaucho contains the active compound, imidacloprid, and other adjuvants. Imidacloprid was found to impart phytotoxicity to lettuce, while the adjuvants were not injurious (F. Tetteroo, personal communication). Therefore, active compounds, not the inert ingredients, primarily cause seed-treatment phytotoxicity. However, seedcoating polymers must be tested for their compatibility and phytotoxicity with each active compound. Seedtreatment compounds should be able to diffuse into large-seeded legume seeds with permeable seed coats as there is no barrier to compound diffusion in this group. In support of this, soybean seeds have been shown to take up herbicides, including amiben, atrazine, linuron and chlorpropham (Rieder et al., 1970), as well as the systemic fungicides benomyl, carboxin and chloroneb (Thapliyal and Sinclair, 1970). The herbicide, 2,4-dichlorophenoxyacetic acid (2,4-D) was absorbed by pea (Pisum sativum) seeds (Hansen and Buchholtz, 1952). Seed-treatment compounds that are non-ionic and moderately lipophilic in nature should be able to diffuse through crop seeds with selective permeability. Thus, phytotoxicity could be induced prior to radicle emergence. In addition, mechanical damage of the onion seed coat allowed diffusion of 
water-soluble compounds from the environment into the embryo (Beresniewicz et al., 1995b).

An anomaly is how seed-treatment actives can pass through non-permeable seed covering layers in cucumber and lettuce. An explanation for cucumber can be found in the discontinuity of the perispermendosperm envelope at the micropylar region of the seed. Coumarin was observed to diffuse at the micropylar region, but not the perisperm-endosperm that surrounded the cotyledons (Salanenka et al., 2009). A micro-canal was observed at the nucellar beak and this anatomical feature was proposed as a conduit allowing diffusion of coumarin to the radicle tip. Similar to cucumber, coumarin was observed in the micropylar area of lettuce embryos, suggesting a discontinuity or weakness in the endosperm envelope in this region (Salanenka and Taylor, unpublished data).

\section{Conclusion}

The use of seed treatments will continue as an integral component of early season pest management, and for the application of active compounds to enhance germination and seedling establishment. A criterion in the development of new seed treatments is that the active compound should not be phytotoxic. However, technologies have been developed to reduce this potential phytotoxicity through the seed-coating system and/or the addition of amendments to the seed-testing medium. The relationship between physico-chemical properties of applied chemicals and seed-coat permeability has been established for ten crop seeds representing seven plant families. A technique is proposed to determine the seed-coat permeability characteristic of any species based on the uptake of two fluorescent tracers. Increased knowledge of seed-coat permeability provides a basis to predict the effectiveness and potential phytotoxicity of active compounds, to develop 'smarter' seedtreatment formulations, and to develop methods to enhance permeability characteristics of selected crop seeds.

\section{Acknowledgements}

The authors thank B. Hamman, H. Hill and F. Tetteroo for providing information and industry perspectives.

\section{References}

AOSA (2009) Rules for testing seeds. Ithaca, New York, Association of Official Seed Analists.

Beresniewicz, M.B., Taylor, A.G., Goffinet, M.C. and Koeller, W.D. (1995a) Chemical nature of a semipermeable layer in seed coats of leek, onion, tomato and pepper. Seed Science and Technology 23, 135-145.

Beresniewicz, M.B., Taylor, A.G., Goffinet, M.C. and Terhune, B.T. (1995b) Seed coat integrity in relation to amino acid leakage in onion and leek. Plant Varieties and Seeds 8, 87-96.

Briggs, G., Bromilow, R. and Evans, A. (1982) Relationships between lipophilicity and root uptake and translocation of non-ionized chemicals by barley. Pesticide Science 13, 495-504.

Copeland, L.O. and McDonald, M.B. (2001) Principles of seed science and technology (4th edition). Dordrecht, Kluwer Academic Publishers.

Edgington, L.V. and Peterson, C.A. (1977) Systemic fungicides: theory, uptake and translocation. pp. 51-89 in Siegel, M; Sisler, H.D. (Eds) Antifungal compounds, Vol. 2. New York, Marcel Dekker.

Halmer, P. (1999) Commercial seed treatment technology. pp. 257-286 in Black, M; Bewley, J.D. (Eds) Seed technology and its biological basis. Sheffield, Sheffield Academic Press.

Halmer, P., Bewley, J.D. and Thorpe, T.A. (1975) Enzyme to break down lettuce endosperm cell wall during gibberellin- and light-induced germination. Nature $\mathbf{2 5 8}$, 716-718.

Hansen, J.R. and Buchholtz, K.P. (1952) Absorption of 2, $4-\mathrm{D}$ by corn and pea seeds. Agronomy Journal 44 , 493-496.

Hill, H.J. (1999) Recent developments in seed technology. Journal of New Seeds 1, 105-112.

Kanampiu, F., Karaya, H., Burnet, M. and Gressel, J. (2009) Needs for and effectiveness of slow release herbicide seed treatment Striga control formulations for protection against early season crop phytotoxicity. Crop Protection 28, 845-853.

Kuhar, T.P., Stivers-Young, L.J., Hoffmann, M.P. and Taylor, A.G. (2002) Control of corn flea beetle and Stewart's wilt in sweet corn with imidacloprid and thiamethoxam seed treatments. Crop Protection 21, 25-31.

Rieder, G., Buchholtz, K.P. and Kust, C.A. (1970) Uptake of herbicides by soybean seed. Weed Science 18, 101-105.

Russell, P.E. and Mussa, A.E.A. (1977) An evaluation of potential seed treatments to control Fusarium solani f.sp. phaseoli, the cause of foot and root rot of Phaseolus vulgaris. Journal of Agriculture Science 89, 235-238.

Salanenka, Y.A., Goffinet, M.C. and Taylor, A.G. (2009) Structure and histochemistry of the micropylar and chalazal regions of the perisperm-endosperm envelope of cucumber seed associated with solute permeability and germination. Journal of the American Society for Horticultural Sciences 134, 479-487.

Salanenka, Y.A. and Taylor, A.G. (2009) Uptake of model compounds by soybean, switchgrass and castor seeds applied as seed treatments. pp. 76-81 in Symposium Proceedings No. 83. Seed production and treatment in a changing environment. Alton, Hampshire, UK, British Crop Protection Council.

Salanenka, Y.A. and Taylor, A.G. (2011) Seedcoat permeability: uptake and post-germination transport of applied tracer compounds. HortScience 46, 622-626.

Simpson, G.M. (1990) Seed dormancy in grasses. Cambridge, Cambridge University Press. 
Taylor, A.G. (2003) Seed treatments. pp. 1291-1298 in Thomas, B.D.; Murphy, J.; Murray, B.G. (Eds) Encyclopedia of applied plant sciences. Kidlington, Oxford, UK: Elsevier Academic Press.

Taylor, A.G., Eckenrode, C.J. and Straub, R.W. (2001) Seed treatments for onions: challenges and progress. HortScience 36, 199-205.

Tetteroo, F., Kofman, F. and Legro, B. (2009) Smart pellet technology for safe and accurate insecticide applications. pp. 43-49 in Symposium Proceedings
No. 83. Seed production and treatment in a changing environment. Alton, Hampshire, UK, British Crop Protection Council.

Thapliyal, P.N. and Sinclair, J.B. (1970) Uptake of the three systemic fungicides by germinating soybean seed. Phytopathology 60, 1373-1375.

Yim, K.O. and Bradford, K.J. (1998) Callose deposition is responsible for apoplastic semipermeability of the endosperm envelope of muskmelon seeds. Plant Physiology 118, 83-90. 